\title{
Performance Analysis of Multi-Channel CR Enabled IoT Network with Better Energy Harvesting
}

\author{
Afiya Kiran ${ }^{1}$, Ahmad Karim ${ }^{2, *}$, Yasser Obaid Alharbi ${ }^{3}$ and Diaa Mohammed Uliyan ${ }^{3}$ \\ ${ }^{1}$ Department of Computer Science, BZ University, Multan, 6000, Pakistan \\ ${ }^{2}$ Department of Information Technology, BZ University, Multan, Pakistan \\ ${ }^{3}$ College of Computer Science and Engineering, University of Ha'il, Hail, Saudi Arabia \\ ${ }^{*}$ Corresponding Author: Ahmad Karim. Email: ahmadkarim@bzu.edu.pk \\ Received: 17 July 2021; Accepted: 23 August 2021
}

\begin{abstract}
Wireless Sensor Networks (WSNs) can be termed as an autoconfigured and infrastructure-less wireless networks to monitor physical or environmental conditions, such as temperature, sound, vibration, pressure and motion etc. WSNs may comprise thousands of Internet of Things (IoT) devices to sense and collect data from its surrounding, process the data and take an automated and mechanized decision. On the other side the proliferation of these devices will soon cause radio spectrum shortage. So, to facilitate these networks, we integrate Cognitive Radio (CR) functionality in these networks. CR can sense the unutilized spectrum of licensed users and then use these empty bands when required. In order to keep the IoT nodes functional all time, continuous energy is required. For this reason the energy harvested techniques are preferred in IoT networks. Mainly it is preferred to harvest Radio Frequency (RF) energy in the network. In this paper a region based multi-channel architecture is proposed. In which the coverage area of primary node is divided as Energy Harvesting Region and Communication Region. The Secondary User (SU) that are the licensed user is IoT enabled with Cognitive Radio (CR) techniques so we call it CR-enabled IoT node/device and is encouraged to harvest energy by utilizing radio frequency energy. To harvest energy efficiently and to reduce the energy consumption during sensing, the concept of overlapping region is given that supports to sense multiple channels simultaneously and help the SU to find best channel for transmitting data or to harvest energy from the ideal channel. From the experimental analysis, it is proved that $\mathrm{SU}$ can harvest more energy in overlapping region and this architecture proves to consume less energy during data transmission as compared to single channel. We also show that channel load can be highly reduced and channel utilization is proved to be more proficient. Thus, this proves the proposed architecture cost-effective and energy-efficient.
\end{abstract}

Keywords: Wireless sensor network; multi-channel sensing; energy harvesting; cognitive radio; IoT network

This work is licensed under a Creative Commons Attribution 4.0 International License, which permits unrestricted use, distribution, and reproduction in any medium, provided the original work is properly cited. 


\section{Introduction}

A wireless sensor network (WSN) is considered as a chief support for monitoring and managing industrial, medical, agriculture, transportation and military applications. A WSN consists of thousands of small and less expensive sensor nodes that interact with each other for the purpose of sensing the environment, process the collected data and communicate to take the appropriate decisions [1].

These small sensor nodes collective are known as Internet of Things (IoT). The IoT is basically a group of sensor devices that are deployed over a large distance or sometimes they are not physically present together. But their aim is to collectively work for a specific purpose. They share their collected information among themselves and co-ordinate with each other in order to perform a specific task. IoT nodes are battery rechargeable devices that are deployed over required coverage areas to make network more efficient [2]. The decision taken by these devices is more effective and timely reducing the risk of damage.

Cognitive radio $(\mathrm{CR})$ can be defined as a smart communication system that collects its surrounding nodes information. From the collected information it can automatically take decision to perform two main functions that are efficient spectrum utilization and reliable communication [3]. $\mathrm{CR}$ works as a decision taker device in the network to control the communication, it senses the licensed channel when it is not used by the Primary User (PU), it handover the channel or sometimes a portion of the spectrum to the Secondary User (SU) for communication or energy harvesting. In Cognitive Radio Network (CRN) the nodes can be categorized as 1) primary users (PUs) that own a specific spectrum and 2) secondary users (SUs) that does not own any spectrum and use the PU channel for communication.

In Ad-hoc CR structure, the IoT nodes are facilitated with all CR capabilities [4]. These $\mathrm{CR}$ enabled IoT devices after observing the surrounding data effectively, take decision through the machine learning algorithms and when many IoT devices are working collaboratively for a common purpose the IoT devices directly communicate with each other after processing data from local environment and do not need any central unit. These IoT nodes also do not require any external assistance to connect and share information to CR-enabled IoT devices of other networks. The major problem faced during maintaining IoT devices is limited energy storage of the nodes [5,6]. Once the battery of devices fade away, the communication will be stopped until the battery would be replaced or by finding any other source of energy. Practically it is almost impossible to change the energy storage time to time. Therefore the advances for energy harvesting from the environment is preferred for long life of nodes in the network [7,8]. The energy can be harvested from the natural sources like solar, thermal, wind, chemical etc or energy sources installed by human beings. Energy can be gained from these sources or energy can be gained from the spectrum $[9,10]$. Radio Frequency (RF) energy harvesting is a processing of converting energy from electromagnetic sources into voltage. In CR enabled IoT network the secondary nodes harvest energy from the primary user spectrums i.e., from the licensed channels. In some networks it is very challenging to provide any other source of energy to the nodes, the energy harvested from solar or other natural sources also could not be enough to keep the nodes alive, nodes battery cannot be changed time to time as it is very expensive to keep the network functional. Thus energy harvested from the RF by utilizing the PU spectrum proves to be more beneficial and reasonable technique to keep the nodes alive and network functional all time.

When the SU is utilizing licensed spectrum the CR constantly monitors the presence or absence of the PU. When a primary user is comes back to use its channel, SU has to leave 
the channel and CR again sense spectrums to find vacant channel in order to continue the transmission. A lot of energy and time is wasted during sensing the channel again and again.

To continue the transmission of the node with minimum delay multi-channel sensing techniques are being focused. Multi-channel sensing in CR solves this problem by sensing multiple channels simultaneously to make continuous transmission of secondary node. Collaborative multichannel sensing efficiently reduce the sensing energy and delay in the communication [11]. In a CR multi-channel architecture, the CR-enabled IoT device sense multiple spectrums simultaneously, process the gathered channel states and decide the channel for SU.

In previous studies the researchers mainly focused to apply IoT system in spectrum sharing $[12,13]$ or enabling IoT devices for energy harvesting. However, there are few studies that focus on spectrum sharing techniques with energy harvesting enabled IoT devices. The corporative information transmission mode is essential for the IoT and the shared spectrum. In most of studies time slot mechanism is used for sensing channel, data communication and energy harvesting [14]. Considering the challenges of the existing spectrum sharing networks, it is necessary that, IoT nodes adept CR advances for spectrum sensing and selecting spectrum for energy harvesting without any external assistance along with region based multi-channel sensing approach to enable the IoT nodes to sense multiple regions simultaneously and to take the efficient decision.

This paper proposes region based multi-channel sensing architecture in ad-hoc CR enabled IoT network. The IoT devices in the network sense multiple regions simultaneously without any external aid as they are enabled with CR techniques. The network comprises of secondary and primary nodes. The coverage area of primary node in divided into Harvesting Region and Communication Region. The SU is allowed to harvest energy when it is in harvesting region and transmit data when it is in communication region. The paper compares the energy harvesting and energy consumption behavior when the SU can sense single channel and when SU can sense multiple channels. Also discuss the pattern of channel utilization, channel load and availability for uni-channel sensing and for the CR-enabled multi-channel sensing architecture.

The rest of the paper discusses the literature behind this work, the proposed architecture, experimental results and discussion on them. And last the conclusion and some future work related to proposed study.

\section{Literature Review}

Cognitive radio is proposed as a novel solution to solve the spectrum deficit problems. The basic idea of cognitive radio is that unlicensed or Secondary Users (SUs) are allowed to utilize the spectrum of licensed users which are also known as primary users (PUs) in an opportunistic manner [15,16]. The concept of CR-based IoT network is briefly discussed in [17], in which the principles of implementing CR in IoT based applications and the schemes and frameworks are explained. The frameworks are classified on the basis of context and volume of data. Lastly, the survey presents the real-world issues and the future directions for hardware devices of CR-based IoT network. But it did not focus on the techniques and ways to share vacant spectrum bands, the MAC protocols and the appropriate spectrum sensing approaches for an IoT based application. A comprehensive study of applying frameworks in spectrum sharing for CR- based IoT networks is proposed in [18]. The study evaluate the existing schemes of spectrum sensing and sharing, the issues and the challenges that are faced in real world applications, and then propose an architecture of spectrum sharing process on four layers in CR-based IoT networks. However, the study did not focus on the suitability of spectrum sensing techniques for IoT applications and the 
adaptation of the proper MAC protocol. Afterwards, a lot of research carried on the approaches that include channel assignments to MAC protocols in wireless networks and cognitive radio networks. A local synchronized and periodic Sensor-MAC (SMAC) protocol presented in [19], Wise-MAC that uses TDMA protocol for data channel assessment and CSMA for control channel assessment in [20], and a MAC protocol SIFT proposed for event driven sensor network in [21].

Due to limited number of channels and uncertain time errors the present multi-channel protocols are not sufficient. The authors in [22] put forward a novel tree-based multichannel scheme TMCP for data collecting applications, the disjoint trees are allocated channels to achieve parallel transmission in the network rather that assigning channels to the nodes. Potential radio interference can be highly reduced by using greedy algorithm. By this technique the performance of the network can be elevated.

When the energy of sensor node is depleted its battery need to be changed which was more challenging and sometimes impossible. There battery-free energy harvesting nodes were more encouraged in the network. The authors in [23] propose an online algorithm for IoT nodes to harvest energy in cognitive radio network without compromising the data quality and transmission delay. The algorithm proves to be cost effective and optimal solution as compared to non-energy harvesting case. In [24] suggest a hybrid energy storage technique which can refill its energy from the environment. The studies shows that single fixed capacitor cannot fulfill the energy harvesting requirements of scattered mobile networks. Hence a dual-capacitor is encouraged in the paper. The environmental flexible learning algorithm anticipates the available ambient energy and shift between two capacitors. Radio frequency energy harvesting techniques are becoming as an emerging technology for advance wireless network. The authors in [25] present a novel architecture where a node utilize the electromagnetic waves from the radio frequency signals emit in wireless network system to harvest its energy consumed during the data transfer. The researchers in [26] propose a CR-WSN network based on energy harvesting model in a non-ordinary Markovian battery structure and also propose the frame structure sensing the spectrum and energy harvesting. In [27] suggest a new networking architecture in which a smart grid environment the IoT devices are empowered with energy harvesting techniques it enhances the compatibility and connectivity of IoT nodes in the network. The proposed architecture launches CR in IoT enabled smart grid to control spectrum shortage problem and creating a more efficient network. Researchers are focusing for designing RF energy harvesting circuits. The architecture presented in [28] focus on a unique approach in which he secondary user made capable to harvest energy from the primary transmitter. The Power transmitter is protected from secondary node interference by relating a guard zone. The secondary node harvest energy in the harvesting zone. Noticing the impact of harvesting and guard zone the transmission probability is being noticed. Early corporative multi channel sensing consumes more energy, to sense the spectrums and decide a vacant spectrum in the network. The authors in [29] applies non-orthogonal multiple access (NOMA) to cognitive orthogonal frequency-division multiplexing (OFDM) architecture in order to enhance the IoT nodes lifetime. To harvest energy from radio signals, power splitting mode is applied. Also two cognitive modes: overlay mode and underlay mode are proposed to enhance the performance of architecture. The researchers in [30] propose an efficient region based multichannel sensing. A winwin architecture is designed according to which a secondary user is allowed to sense the spectrum. If the channel is found idle secondary user transmit data and if the channel is found busy the SU can utilize the channel for energy harvesting. Only a selective number of nodes are allowed 
to sense the spectrum in order to reduce the sensing energy. By using machine learning algorithm SUs can be efficiently trained to sense and take the decision.

\section{Proposed Architecture}

For a simple ad-hoc network we consider that there are two primary nodes P1 and P2 and a secondary node SU. Usually a primary node covers $38 \mathrm{~km}$ circular area. The coverage area of primary node is divided into two regions; the near circular area 0-19 km specified as energy harvesting region, and $19-38 \mathrm{~km}$ circular area as communication region. A SU is permitted to harvest energy when it is in harvesting region and allowed to transfer data when it is in communication region. The regions of nearby nodes overlap with each other giving the concept of multichannel sensing for a SU. The paper emphasis on the behavior of secondary node in these overlapped regions. The energy harvesting and data communication behavior when SU is in coverage area of one primary node and when it is in overlap regions. The proposed network is shown in Fig. 1.



Figure 1: Proposed architecture

In this proposed architecture $\mathrm{P} 1$ is the first primary node, $\mathrm{P} 2$ is the second primary node, $\mathrm{R} 11$ is the harvesting region of node $\mathrm{P} 1, \mathrm{R} 12$ is communication region of $\mathrm{P} 1, \mathrm{R} 21$ represents harvesting region $\mathrm{P} 2$ and $\mathrm{R} 22$ as communication region of P2. These regions overlap in three ways; a) the harvesting region of nodes overlap with each other b) the communication region of both nodes overlap and c) harvesting region of one node overlap with communication region of other node. The co-ordinates of primary nodes are assumed to be known by using GPS tracker. The coordinates of SU (x, y) are estimated by Angle of Arrival (AoA). The AoA uses triangulation principle to find the position of the node. 
The primary nodes have different Antenna Gains. The antenna gain can be defined as the power of the node. It expresses the amount of energy harvested from harvesting region and energy consumed during communication. The gained power can be calculated by the Friis Equation.

The algorithm for proposed network is divided into two halves, when there is Uni-Channel Sensing i.e., SU is in single region and when SU is in overlap region and performs Multi-Channel Sensing.

\section{Algorithms}

\subsection{Uni-Channel Sensing}

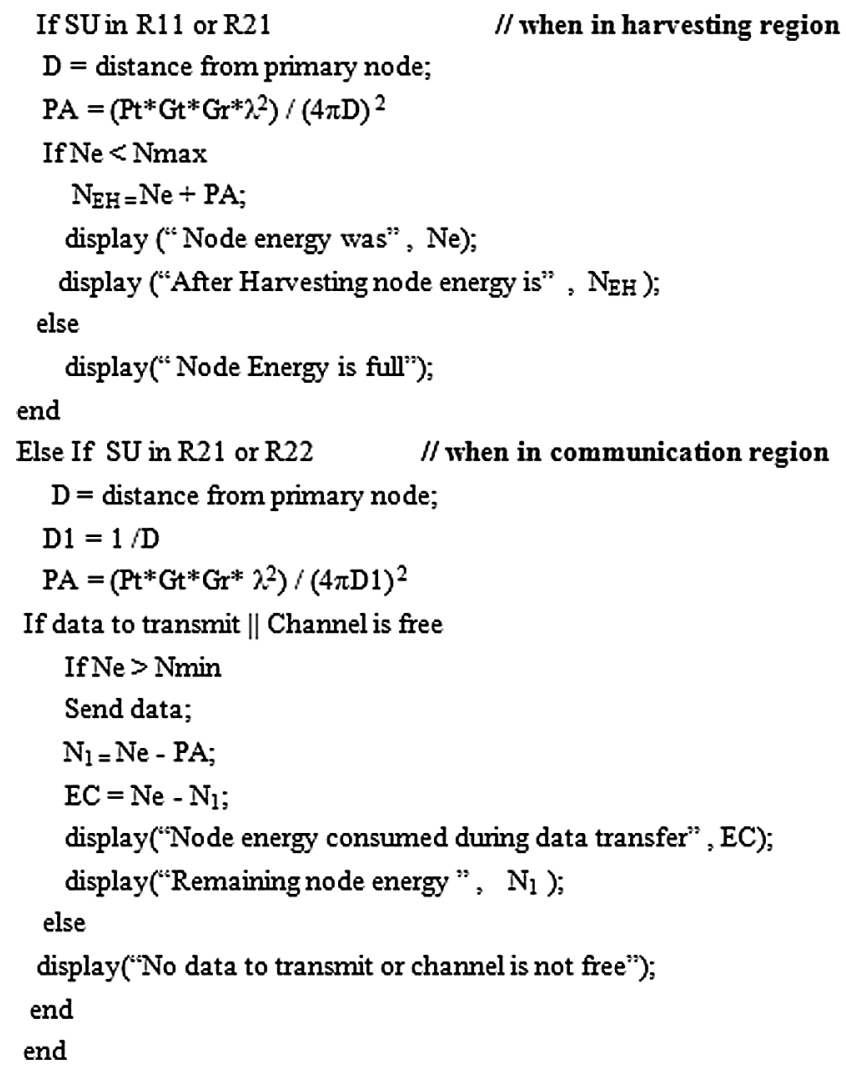

In single channel sensing if $\mathrm{SU}$ is in energy harvesting region of a node, it calculates the distance from that node by using Euclidean distance formula and checks whether there is need of energy or not. If node energy is not full, it harvest from the primary node. The quantity of energy harvested depends on antenna gain of the primary node.

Similarly if SU is in communication region of any node it determines whether SU has any data to be transmitted and the PU channel is free, and node energy is sufficient for transmission, the SU transmit the data from that licensed channel. 


\subsection{Multi-Channel Sensing}

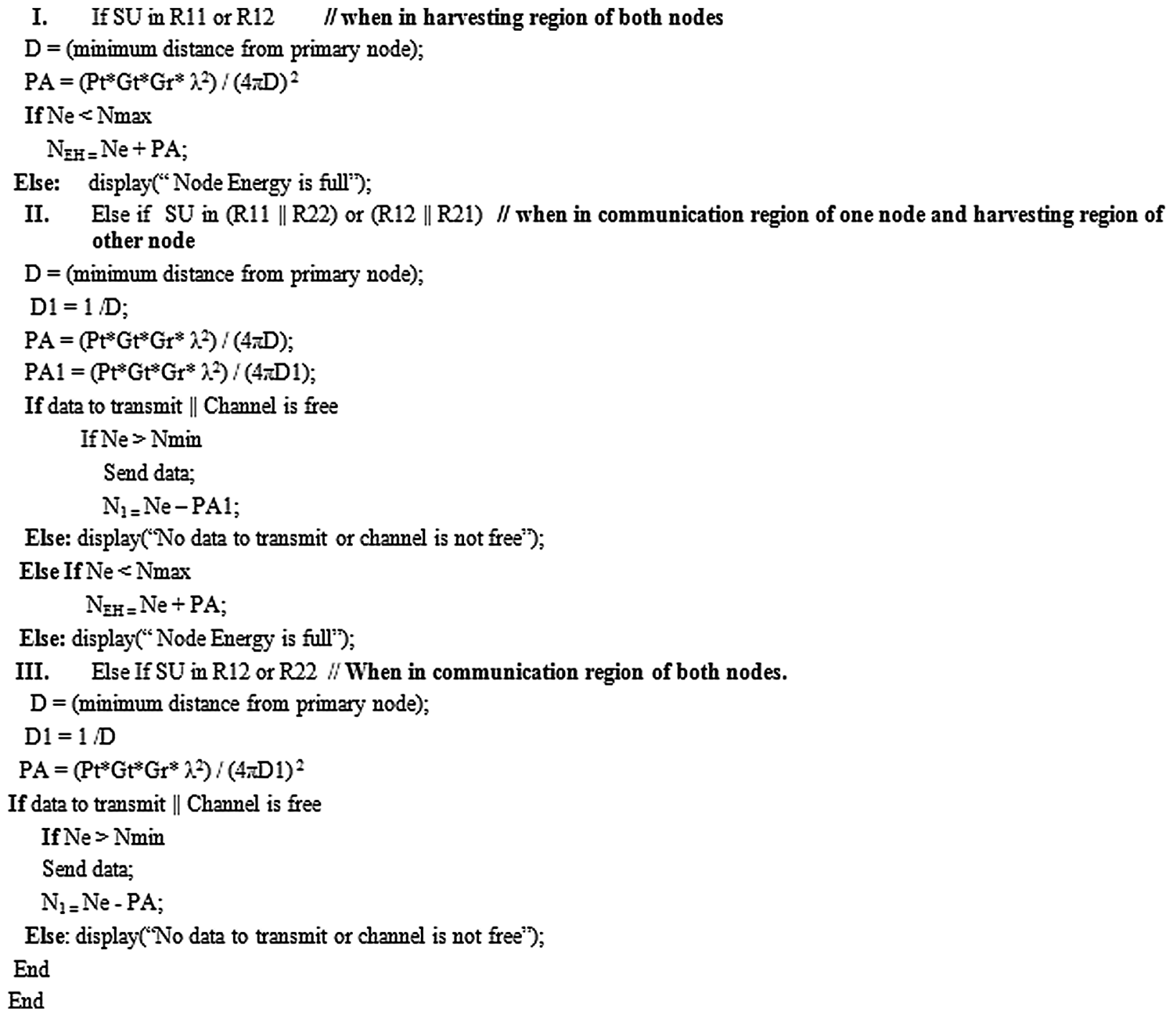

In Multi-Channel sensing when the CR based SU is in multiple regions (overlap region) of nodes; choose the operation on the basis of following three conditions:

(a) If the SU is in harvesting region of both nodes, the SU determines whether there is any need of harvesting and then harvest from the nearest primary node.

(b) If the SU is in the harvesting region of one node and in the communication region of other node, CR checks out if $\mathrm{SU}$ has some data for transmission, licensed channel is unoccupied and SU energy is enough for communication, it permits SU to use the channel for communication. If any these conditions didn't fulfils it checks the energy level of SU, if there is need to harvest energy it allows SU for harvesting. 
(c) If node is in communication region of both primary nodes, the node uses the channel of nearest primary node if there is any data for transmission and node energy is enough for the communication. Also if the licensed channel is unoccupied.

The proposed algorithm assists the SU to sense multiple channels simultaneously on the basis of regions and take proficient decision according to the requirement of the node. This makes network more dedicated and exceptional.

\section{Results and Discussion}

The experiment is performed in MATLAB R2019b for experiment a licensed channel of $915 \mathrm{~Hz}$ frequency is considered. Initial node energy is $60 \mathrm{dBm}$ (900 joules). Maximum node energy assumed to be $1.0^{15}$ joules and threshold value or minimum energy required for data transmission should be $1.0^{10}$ joules. If $\mathrm{SU}$ is near to the PU more energy can be harvested and as the SU moves away the amount of energy harvesting decreases. Similarly if node is near to PU less energy is consumed during data transmission and as the distance increases the amount of energy consumption also increases. The maximum and minimum energy that a SU can harvest or consumed during communication can be;

Tab. 1 shows that node can harvest more energy when is near to primary node P2 than P1. And maximum energy consumed during transmission is less when SU communicate through P1 channel as compared to P2. So for a more efficient network SU should prefer P2 for energy harvesting and P1 for data transmission.

Table 1: Maximum and minimum energy harvested or consumed

\begin{tabular}{lllll}
\hline Node & $\begin{array}{l}\text { Max-energy } \\
\text { harvested }\end{array}$ & $\begin{array}{l}\text { Min-energy } \\
\text { harvested }\end{array}$ & $\begin{array}{l}\text { Max-energy } \\
\text { consumed }\end{array}$ & $\begin{array}{l}\text { Min-energy } \\
\text { consumed }\end{array}$ \\
\hline P1 & $5.1^{11}$ & $1.4^{09}$ & $3.7^{14}$ & $2.2^{14}$ \\
P2 & $1.63^{12}$ & $1.6^{12}$ & $1.1^{15}$ & $7.2^{14}$ \\
\hline
\end{tabular}

\subsection{Energy Harvesting Phenomenon}

The primary node $\mathrm{P} 2$ has more energy transmitting power than the primary node $\mathrm{P} 1$. So more energy is harvested when SU harvest from P2 than P1.

The rise in energy level when harvested from P1 and P2 is shown in Fig. 2.

Fig. 2 represents that as SU moves toward the PU there is increase in energy level. When the $\mathrm{SU}$ is at maximum distance $17 \mathrm{~km}$ away from the primary nodes, the energy harvested from $\mathrm{P} 1$ is $1.7^{09}$ joules and energy harvested from P2 is $5.6^{09}$ joules, there is not much great difference in energy harvesting when SU is away from the node. But when a SU is nearest to PU at distance of $5 \mathrm{~km}$ then energy harvested from P2 is $6.5^{10}$ joules and from P1 is $2.0^{10}$ joules, hence the energy harvested from P2 is much more than P1.

In order to make network more proficient the proposed architecture allow SU to harvest energy from the nearest node. In overlap region when node is close to P1 it harvest energy from P1 and when it is near to P2 it harvest energy from P2. Hence the energy harvested in overlap region is more efficient than energy harvested from node P1 only.

The energy harvesting phenomenon from P1 and overlap region is shown in Fig. 3. 




Figure 2: Energy harvested from P1 and P2

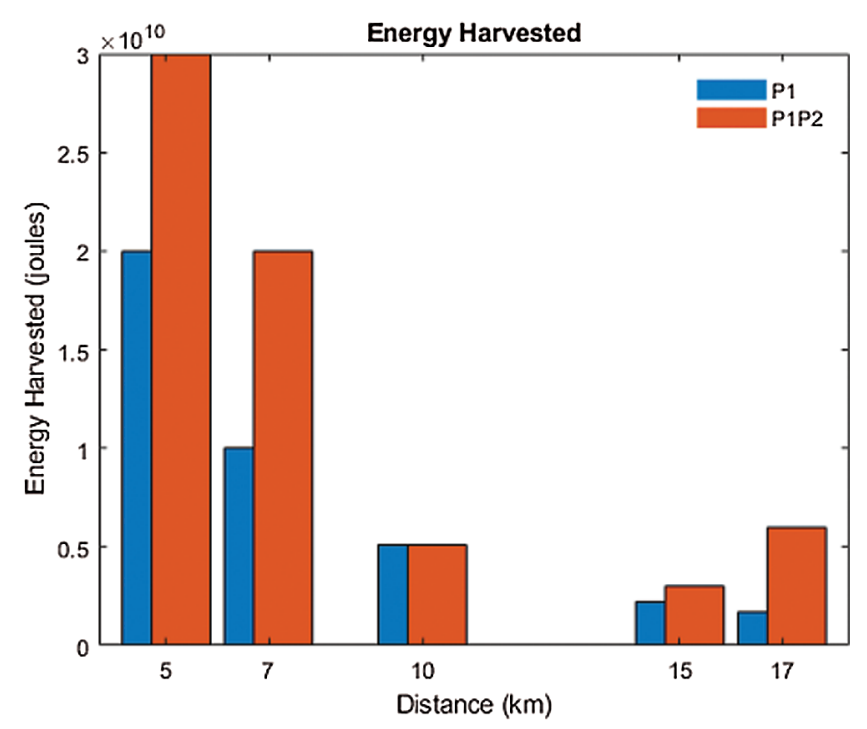

Figure 3: Energy harvested from $\mathrm{P} 1$ and $\mathrm{P} 1 \mathrm{P} 2$

Fig. 3 represents that SU harvest more energy from overlap region as compared to node P1. More time will consume if node refills energy from node P1 than the overlap region. Hence it is focused that when SU is in harvesting region of both primary nodes i.e., in overlap region, it should harvest energy from its closest primary node in contrast to that it harvest energy from a single primary node.

\subsection{Energy Consumption Phenomenon}

The ratio of energy consumed for communication through licensed channel depends on data size and mainly on the antenna power of the primary node. A PU with more antenna gain 
consumes more energy during data transmission as compared to a PU that has low antenna gains. Also if SU is away from the PU more energy is drained. In proposed network P1 takes less energy during data transmission because it has less antenna gains as compared to P2 that consumes more energy for transmission as its antenna gains are high. It is focused that least energy should be consumed during data transmission so that SU could communicate for long time.

Fig. 4 shows this fact,



Figure 4: Energy consumption of P1 and P2

It is shown in Fig. 4 that as the SU moves away that is distance increases from the primary node, the energy required for data transmission also increases.

The primary node P2 consumes more energy because of high antenna gain than the P1 that has less antenna gains. In order to make the network more consistent and energy proficient the model of overlap region is presented. In overlap communication region when the SU is near to P1 it transmits data through P1 channel and when is near to P2 it communicates data through P2. In this way network consumes less energy in contrast to the situation when it only transmits data through the node that has antenna gains.

This fact is shown in Fig. 5.

Fig. 5 represents that in overlap region (communication region of both nodes) the decision is taken on the basis of distance, the SU communicates from the channel to the nearest primary node. In experiment during 6 time slots, three times the SU is near to P1 and transmit data utilizing its channel and three times SU is near to P2 and uses its channel. But still the accumulative energy consumed in overlap region is much less than the energy consumed if data is only transmitted from node P2. Thus with the help of overlap region energy consumption of a node can be significantly reduced as compared the data only transmitted from the node that requires more transmitting power. In this way the network proves to be more cost-effective. Thus with the concept of overlap regions there is a prominent change in energy harvesting and energy consumption as compared to the data transmitted or energy harvested from a single primary node. 


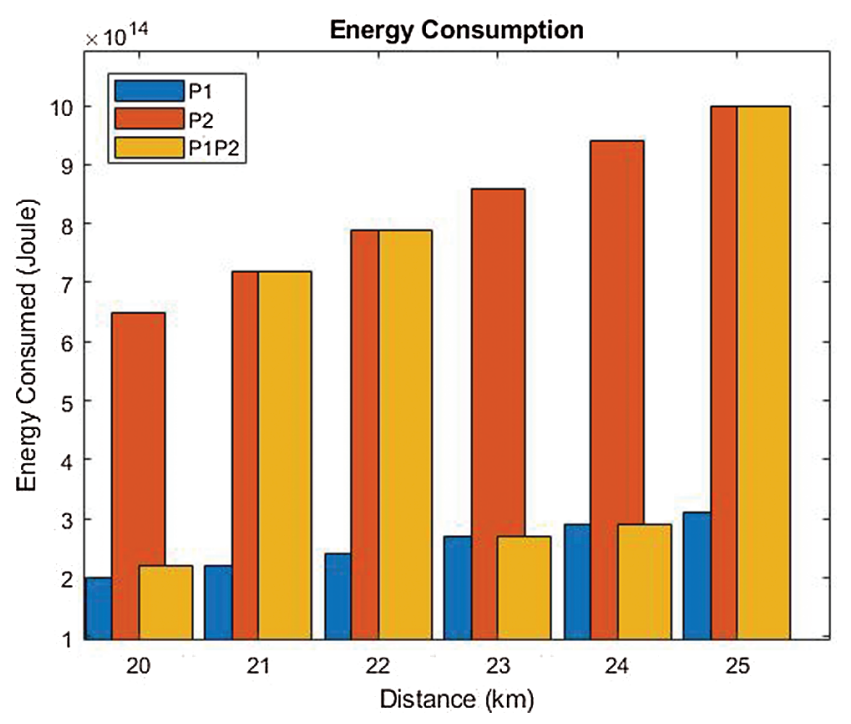

Figure 5: Energy consumption phenomenon

\subsection{Channel Utilization}

Channel utilization can be described as the portion of time available for SU to use the licensed channel, until PU arrives back to use its channel. If there are $\mathrm{N}$ numbers of SU, and the channel is allotted to yth preferred SU.

The channel utilization can be calculated as Eq. (1).

$C U=\frac{(N+1)-y}{N}$

The Eq. (1) is applied when there is one channel available for a SU or SU can sense and takeover only one channel at a time.

When SU are authorized with CR functionalities and can sense more than one channel in the network, channel utilization can be calculated as Eq. (2).

$C U=\frac{\sum_{m=1}^{M} \wedge(N+1)-y \times m}{N \times M}$

When the SU can sense single channel and takeover that channel for transmission the SU that channel for more time even it is not much preferred and is away from the SU. But when the SU is empowered with CR abilities, SU sense multiple channels then SU utilizes a less preferred channel for less time and then takeover other preferred channel as soon as possible.

Fig. 6 shows channel utilization when there is single channel and when SU is in overlap region giving the concept of multi-channel.

In Fig. 6, there is preference of SU to use the primary channel on $\mathrm{x}$-axis and utilization rate on y-axis. Channel preference shows how much SU prefers this channel to be used. Channel utilization is maximum when the channel is first priority of SU, and decreases as the priority decreases. Blue curve shows channel utilization when the SU can sense and use only channel, and red curve shows the channel utilization when the $\mathrm{SU}$ is enabled to sense multiple channels. 


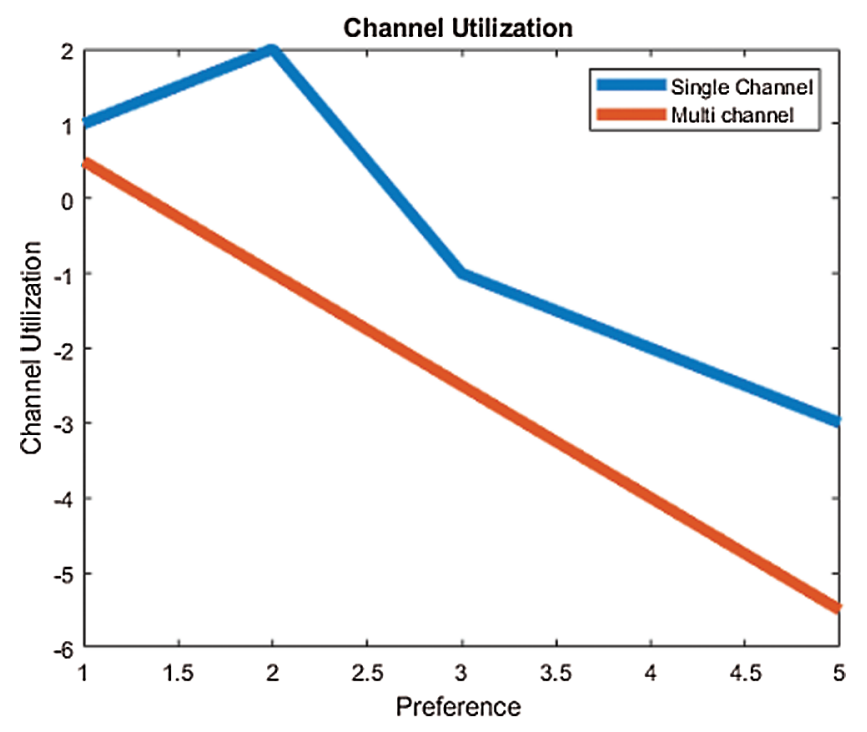

Figure 6: Channel utilization

Fig. 6 shows that channel utilization of overlap region is less than the single channel, it means that as the preference of SU decreases the channel is allocated for small time and as soon as new available channel is sensed the SU is shifted to that channel. And when there is an only one channel available channel utilization decrease slowly. This proves that overlap regions intends to provide better channel assignment to the secondary channel as compared to the single region.

\subsection{Channel Load}

Channel load represents the proportion of licensed channel used by the SU in a specific time. The data traffic or the number of users that attempts to use the primary channel described as channel load. The channel load can be calculated as the Eq. (3).

$C L=\frac{\lambda}{\mu \times C}$

In Eq. (3), $\lambda$ is the arrival rate, $\mu$ is service rate and $\mathrm{C}$ represents the number of channels.

The channel load for a single channel and multi-channels are shown below.

In Fig. 7, arrival rate is shown on x-axis and load is represented on y-axis. The blue line represents the channel load when there is only one channel that is SU can sense and use a single channel and red line shows when the SU is in overlap region and can sense multiple channels simultaneously. This figure shows that as the arrival rate increases channel load also increases. When the arrival rate is minimum 5 the load on single channel is 1 and for multi-channel is 0.5. And when the arrival rate is maximum i.e., 25 the load is 5 for single channel and 2.5 for the multi-channel scheme. This shows that when the SU is CR-enabled and is in overlap region, the channel load can be reduced effectively. When there are more secondary users in an overlap region the requests of SU can be divided among the channels, which greatly reduces the channel load and enhances the channel availability. This approach makes the network more reliable and optimistic. 




Figure 7: Channel load

The result shows that when SU is enabled with CR techniques it takes more smart decisions as compared when it can sense only single channel. In overlap region the more energy can be harvested as compared to the primary node that has less antenna gain, likewise energy consumption is less other than the node with high antenna gains. The channel utilization and channel load results also proves this architecture to be more proficient and coherent.

\section{Conclusions and Future Work}

In this paper a region based multichannel sensing architecture in a cognitive radio based IoT network is proposed. To improve the network performance it is focused to harvest more energy and to reduce the energy consumption during data transmission by empowering SU to sense multiple channels in a region based overlap region. The experimental results demonstrate that energy harvested from an overlap region is much more than the amount of energy harvested from a single PU having low power transmission. Likewise the energy consumption of node during data transmission can be remarkably reduced if the SU is allowed to communicate from overlap region apart from it communicate from a single PU spectrum with high antenna gains. It is also proved by the experiment that the channel utilization results are more appreciative when the SU is in overlap region because this approach ensures to find best channel for the SU to harvest energy or to send data from the channel. Also the channel load can be remarkable reduced in overlap region as compared to the single channel.

Thus the region based multi-channel sensing technique in CR-enabled IoT network, is proved to be more systematic and well planned as compared to an ordinary network. By this architecture node can harvest more energy in less time, energy consumption during data transmission can be highly reduced, channel utilization is more powerful and load on a channel can be remarkably reduced. Thus this architecture proves to be more cost-effective and energy-saving forming the network more promising and functional.

The experiment is conducted considering two primary users and one secondary user. In future the behavior of network can be studied by considering more PUs and SUs. The overlapped region 
can be expanded and energy saving techniques can be applied. This approach can be implemented in all IoT enables cognitive radio networks.

Funding Statement: The authors received no specific funding for this study.

Conflicts of Interest: The authors declare that they have no conflicts of interest to report regarding the present study.

\section{References}

[1] V. K. Garg, "An overview of wireless systems," in Wireless Communications Networking, I ${ }^{\text {st }}$ ed., vol. 123735807, Morgan Kaufmann, Elsevier, 2007.

[2] A. Almasoud, "Optimizing performance and energy efficiency of group communication and internet of things in cognitive radio networks," Ph.D dissertation. Iowa State University, US, 2018.

[3] S. Haykin, "Cognitive radio: Brain-empowered wireless communications," IEEE Journal on Selected Areas in Communications, vol. 23, no. 2, pp. 201-220, 2005.

[4] I. F. Akyildiz, W. Y. Lee and K. R. Chowdhury, "CRAHNs: Cognitive radio ad hoc networks," $A d$ Hoc Networks, vol. 7, no. 5, pp. 810-836, 2009.

[5] A. H. Sodhro, S. Pirbhulal and A. K. Sangaiah, "Convergence of IoT and product lifecycle management in medical health care," Future Generation Computer Systems, vol. 86, no. 1, pp. 380-391, 2018.

[6] A. S. Adila, A. Husam and G. Husi, "Towards the self-powered Internet of Things (IoT) by energy harvesting: Trends and technologies for green IoT," in 2018 2nd Int. Sym. on Small-scale Intelligent Manufacturing Systems (SIMS), Cavan, Ireland, pp. 1-5, 2018.

[7] P. Kamalinejad, C. Mahapatra, Z. Sheng, S. Mirabbasi, V. C. Leung et al., "Wireless energy harvesting for the Internet of Things," IEEE Communications Magazine, vol. 53, no. 6, pp. 102-108, 2015.

[8] H. Zhang, S. Huang, C. Jiang, K. Long, V. C. Leung et al., "Leung etal, Energy efficient user association and power allocation in millimeter-wave-based ultra dense networks with energy harvesting base stations," IEEE Journal on Selected Areas in Communications, vol. 35, no. 9, pp. 1936-1947, 2017.

[9] J. A. Paradiso and T. Starner, "Energy scavenging for mobile and wireless electronics," IEEE Pervasive Computing, vol. 4, no. 1, pp. 18-27, 2005.

[10] I. A. Ieropoulos, J. Greenman, C. Melhuish and J. Hart, "Comparative study of three types of microbial fuel cell," Enzyme and Microbial Technology, vol. 37, no. 2, pp. 238-245, 2005.

[11] S. Althunibat, T. M. Vuong and F. Granelli, "Multi-channel collaborative spectrum sensing in cognitive radio networks," in 2014 IEEE 19th Int. Workshop on Computer Aided Modeling and Design of Communication Links and Networks (CAMAD), Athens, Greece, pp. 234-238, 2014.

[12] K. Zaheer, M. Othman, M. H. Rehmani and T. Perumal, "A survey of decision-theoretic models for cognitive Internet of Things (CIoT)," IEEE Access, vol. 6, pp. 22489-22512, 2018.

[13] S. Solanki, P. K. Sharma and P. K. Upadhyay, "Adaptive link utilization in two-way spectrum sharing relay systems under average interference-constraints," IEEE Systems Journal, vol. 12, no. 4, pp. 34613472, 2017.

[14] H. Gao, S. Zhang, Y. Su and M. Diao, "Energy harvesting and information transmission mode design for cooperative EH-abled IoT applications in beyond 5G networks," Wireless Communications and Mobile Computing, vol. 2020, no. 8, pp. 1-17, 2020.

[15] A. Goldsmith, S. A. Jafar, I. Maric and S. Srinivasa, "Breaking spectrum gridlock with cognitive radios: An information theoretic perspective," Proc. of the IEEE, vol. 97, no. 5, pp. 894-914, 2009.

[16] A. Alsharoa, H. Ghazzai and M.-S. Alouini, "A genetic algorithm for multiple relay selection in twoway relaying cognitive radio networks," in 2013 IEEE 78th Vehicular Technology Conf. (VTC Fall), Las Vegas, NV, USA, pp. 1-5, 2013. 
[17] A. A. Khan, M. H. Rehmani and A. Rachedi, "Cognitive-radio-based internet of things: Applications, architectures, spectrum related functionalities, and future research directions," IEEE Wireless Communications, vol. 24, no. 3, pp. 17-25, 2017.

[18] H. Kour, R. K. Jha and S. Jain, "A comprehensive survey on spectrum sharing: Architecture, energy efficiency and security issues," Journal of Network and Computer Applications, vol. 103, no. 1, pp. 29-57, 2018.

[19] W. Ye, J. Heidemann and D. Estrin, "Medium access control with coordinated adaptive sleeping for wireless sensor networks," IEEE/ACM Transactions on Networking, vol. 12, no. 3, pp. 493-506, 2004.

[20] C. C. Enz, A. El-Hoiydi, J.-D. Decotignie and V. Peiris, "WiseNET: An ultralow-power wireless sensor network solution," Computer, vol. 37, no. 8, pp. 62-70, 2004.

[21] L. Bao and J. Garcia-Luna-Aceves, "A new approach to channel access scheduling for ad hoc networks," in Proc. of the 7th Annual Int. Conf. on Mobile Computing and Networking, New York, USA, pp. 210-221, 2001.

[22] Y. Wu, K. S. Liu, J. A. Stankovic, T. He and S. Lin, "Efficient multichannel communications in wireless sensor networks," ACM Transactions on Sensor Networks (TOSN), vol. 12, no. 1, pp. 1-23, 2016.

[23] S. P. Lad, V. P. Kulkarni and R. Joshi, "An online algorithm for energy harvesting cognitive radio IoT network," in 2019 IEEE Int. Conf. on Advanced Networks and Telecommunications Systems (ANTS), Goa, India, pp. 1-6, 2019.

[24] B. Munir and V. Dyo, "On the impact of mobility on battery-less RF energy harvesting system performance," Sensors, vol. 18, no. 11, pp. 3597, 2018.

[25] L. Tang and C. Guy, "Radio frequency energy harvesting in wireless sensor networks," in Proc. of the 2009 Int. Conf. on Wireless Communications and Mobile Computing: Connecting the World Wirelessly, Leipzig, Germany, pp. 644-648, 2009.

[26] H. H. Mahmoud, H. M. ElAttar, A. Saafan and H. ElBadawy, "Optimal operational parameters for $5 \mathrm{G}$ energy harvesting cognitive wireless sensor networks," IETE Technical Review, vol. 34, no. sup1, pp. 62-72, 2017.

[27] M. Ozger, O. Cetinkaya and O. B. Akan, "Energy harvesting cognitive radio networking for IoTenabled smart grid," Mobile Networks and Applications, vol. 23, no. 4, pp. 956-966, 2018.

[28] S. Lee, R. Zhang and K. Huang, "Opportunistic wireless energy harvesting in cognitive radio networks," IEEE Transactions on Wireless Communications, vol. 12, no. 9, pp. 4788-4799, 2013.

[29] Z. Na, X. Wang, J. Shi, C. Liu, Y. Liu et al., "Joint resource allocation for cognitive OFDM-NOMA systems with energy harvesting in green IoT," Ad Hoc Networks, vol. 107, no. 12, pp. 102221, 2020.

[30] A. Alsharoa, N. M. Neihart, S. W. Kim and A. E. Kamal, "Multi-band RF energy and spectrum harvesting in cognitive radio networks," in 2018 IEEE Int. Conf. on Communications (ICC), Kansas, MO, USA, pp. 1-6, 2018. 